\title{
Predictors of improvement following early exercises rehabilitation program for patients undergoing open lumbar discectomy
}

\author{
Rasha Ali Ahmed Abdelmowla*1, Rania M. Gamal ${ }^{2}$, Mirette M. Aziz ${ }^{3}$, Walid A. Abdel Ghany ${ }^{4}$, Roshdy Elkhayat ${ }^{5}$ \\ ${ }^{1}$ Medical-Surgical Nursing Department, Faculty of Nursing, Assiut University, Egypt \\ ${ }^{2}$ Rheumatology, Rehabilitation and Physical Medicine Department, Faculty of Medicine, Assiut University, Egypt \\ ${ }^{3}$ Public Health and Community Medicine Department, Faculty of Medicine, Assiut University, Egypt \\ ${ }^{4}$ Neurosurgery Department, Faculty of Medicine, Ain Shams University, Egypt \\ ${ }^{5}$ Neurosurgery Department, Faculty of Medicine, Assiut University, Egypt
}

Received: December 15, 2019

Accepted: February 10, 2020

Online Published: March 26, 2020

DOI: $10.5430 /$ jnep.v10n6p86

URL: https://doi.org/10.5430/jnep.v10n6p86

\begin{abstract}
Background and aims: Low back pain is mostly due to disc herniation and has a burden upon economy and social aspects of life. Failure to improve after open lumbar discectomy is frustrating. Therefore, identifying predictors of improvement is of great clinical benefit. Aims: This research was conducted to evaluate whether an early exercises rehabilitation program using educational booklet would provide benefit to patients following open lumbar discectomy and determine potential factors of improvement.

Patients and methods: Design: Single blind randomized controlled trial. Eighty-eight patients scheduled for open lumbar discectomy from January 2017 to January 2019 at Assiut and Ain Shams Universities hospitals in Egypt were randomly assigned to two groups. Control group $(n=44)$ received routine postoperative instructions while intervention group $(n=44)$ received routine instructions in addition to early exercises rehabilitation program and were also provided with a specifically designed educational booklet. Patients were followed up after six months using Oswestry disability index.

Results: There was significant improvement among intervention group as compared to control group in several domains of Oswestry disability index (walking, sitting, standing, sleeping, travelling and sexual and social life). Better pre-operative Oswestry disability index score and early application of exercises rehabilitation program using an educational booklet predicted better postoperative Oswestry disability index score after six months.

Conclusion: Application of an early exercise rehabilitation program and providing patients with a specifically designed educational booklet would be helpful for patients following open lumbar discectomy. Clinical Relevance: Early exercises rehabilitation program can be used by nursing staff as a reference in management of patients following open lumbar discectomy.
\end{abstract}

Key Words: Low back pain, Lumbar disc herniation, Open lumbar discectomy, predictors of improvement, Early exercises rehabilitation program, Nursing, Educational booklet

\section{INTRODUCTION}

Low back and leg pain caused by lumbar disc herniation is important public health problems due to their high preva- lence, being leading causes of functional impairment and disability and the associated health care expenditure. ${ }^{[1,2]}$ The ability to perform essential daily living activities as sitting,

*Correspondence: Rasha Ali Ahmed Abdelmowla; Email: rashaali249@yahoo.com; Address: Faculty of Nursing, Assiut University, Egypt. 
walking, driving, sleeping, bending or lifting is considered as a fundamental component of independence and is predictive of high quality of life. ${ }^{[2]}$ Lumbar disc herniation is common and usually presented with low back and leg pain and sometimes associated with serious neurologic symptoms due to compression on a nerve root or cauda equina. Lumbar discectomy is the main intervention for lumbar disc herniation. ${ }^{[3]}$

Open lumbar discectomy is usually indicated when diagnostic imaging reveals lumbar disc herniation that correlates with radicular as well as neurological symptoms. Additionally, it is considered the treatment of choice for low back pain when conservative treatment fails. ${ }^{[4]}$

Rehabilitation focusing on stretching and strengthening exercises of muscles surrounding the lumbar spine can be very effective in reducing and preventing pain and instability. Following open lumbar discectomy, it is recommended to start an extensive exercise rehabilitation program to regain full range of motion and strength of the spine, help the return to previous lifestyle, and prevent future problems with spine. ${ }^{[5]}$

Nurses should educate, train, and instruct patients with open lumbar discectomy regarding exercises to be done as long as they feel comfortable. It is recommended to continue exercise program and follow up for at least six months on a regular basis. ${ }^{[6]}$ Patients' education is considered an important role of nurses in health care settings. Rehabilitation nurses can assist patients to attain and maintain maximum function by implementing the recommended treatment strategies and educating patients about the required self-care that could enhance their physical, psychosocial, and spiritual health. ${ }^{[7]}$

There are persistent controversies about the management strategies after open lumbar discectomy. Pain may persist due to muscular atrophy, which develop secondary to longstanding inactivity. ${ }^{[8]}$ Thirty to seventy percent of patients experience residual pain, three to twelve percent seek reoperation, and only seventy percent are able to resume work twelve months after surgery. ${ }^{[9]}$ All patients should receive further management, especially those who still suffer from persisting symptoms following lumbar discectomy. Furthermore, the necessity and duration of restricting activity following lumbar discectomy is controversial. ${ }^{[10]}$

Educational materials promote the ability of patients who suffered from persistent or recurrent back pain to selfmanagement. Education supports patients and helps them to perform the recommended exercises program for low back pain. Stronger integration between the theoretical components of patients education and performing the individualized exercises enhance the possibility of success with intervention.

Published by Sciedu Press
Patients complain of back pain are capable to understand and manage their pain through knowledge concerning pain being influenced by physical activity and exercise, positive nonfearful experience with motion and exercise, staying active and adapting activities, thereby changing back pain beliefs and fear of motion. ${ }^{[11]}$

To date, few studies, and almost none in Egypt, have assessed the effect of postoperative early rehabilitation interventions on patients' functional performance. In this study, we aimed to evaluate whether an early exercises rehabilitation program using educational booklet would provide additional benefit to patients who undergo open discectomy for lumbar disc herniation. Moreover, we sought to determine the predictive factors of improvement following early exercises rehabilitation program for patients undergoing open lumbar discectomy. We hypothesized that our designed early exercises rehabilitation intervention could be a predictor of improving postoperative pain.

\section{Patients ANd methods}

The study is a single blind randomized controlled trial. It was conducted by corporation between the Departments of Medical-Surgical Nursing, Neurosurgery and Physical Medicine and Rheumatology and Rehabilitation at two Universities in Egypt (Assiut and Ain Shams).

Sample size was calculated using G power software as 84 cases, 42 per group. We calculated the sample size for testing the differences between two independent means ( 2 tailed). We used an effect size of $0.8, \beta$ error of 0.05 and power $95 \%$.

Newly diagnosed patients with single level lumbar disc prolapse scheduled for open lumbar discectomy were recruited from the Neurosurgery Departments at Assiut and Ain Shams Universities hospitals. All eligible cases who agreed to participate in the study for duration of two years, from January 2017 to January 2019, were included.

Patients were considered eligible for the study by clinical and radiological assessment. The inclusion criteria included having symptoms and signs of disc protrusion such as predominant symptoms in lower extremities, radicular pain, positive signs of adverse nerve-root tension and restricted straight-leg raise. Diagnosis of all cases was confirmed by magnetic resonance imaging test. Patients with multiple levels lumbar disc prolapse, past history of previous lumbar spine surgery and whose age was below 18 or above 65 years old were excluded. Other exclusion criteria included a concurrent lower extremity pathology (other than that associated with low back and lower extremity pain associated with single level disc injury), cognitive dysfunction, evidence of spinal cord compression, neurological disorders, uncontrolled hy- 
pertension, uncontrolled cardiovascular disease, rheumatic joint disease, pregnancy or peripheral vascular disease with sensory loss at foot.

To continue in the study, the primary post-operation inclusion criterion was that participants should undergo a single level (L3-4, L4-5, or L5-S1) open discectomy without any adverse events (4-6 weeks) following surgery.

Ninety out of one-hundred and sixteen patients were invited to participate (90/116) met the eligibility criteria and gave informed consent to participate in our study. Only two patients dropped out during the follow up due to missed follow up appointments.

Patients randomly allocated into two equal groups using a computer based selection program as group I [Intervention group] and group II [Control group] with ratio of 1:1 assignment.

Outcome staff were blinded to treatment. To avoid selection bias, the researcher who performed the clinical pain assessments and who had access to, and the responsibility to record patients' efficacy data was separated from those carrying out the education of a designed booklet. Results from assessments at baseline were recorded, sealed in an envelope and not disclosed to any site staff apart from the physician who performed it. Another physician did the follow up clinical assessment at first week and at six months after education of a designed booklet.

The control group received the routine care and precautions (having the prescribed medication, information on how to move out of bed, sleep on flat and hard surface and avoid lifting heavy objects), while the intervention group received additionally an early exercises rehabilitation program, as described below, and were provided with an educational booklet. Both groups were followed up for six months postoperatively.

\section{Early exercises rehabilitation program:}

\section{Early postoperative period}

Precautions during this period concerned sleeping, getting in and out of bed, sitting, standing and up and down stairs by a cane. Exercises included breathing, circulation, and early stretching and strengthening exercises (buttock squeeze and abdominal tightening) done within 0-2 weeks after surgery, three times a day. On the contrary, the control group received only routine instructions and precautions concerned sleeping, getting in and out of bed, sitting and standing during this period.

\section{Follow up period}

Precautions regarding back brace, sleeping/lying position, bathing, housework/yard work, driving, leisure and recreation, returning to work and sexual activity were provided. Patients were advised to perform stretching and strengthening exercises within the 4-6 weeks postoperatively three times a day. These exercises included knee to chest, hamstring stretch, calf stretch, partial squat, upper back strengthening, ankle strengthening and standing hip extension. On the contrary, the control group received only routine instructions and precautions concerned sleeping/lying position, housework/yard work and returning to work during this period.

The medical-surgical nursing researcher introduced a copy of the educational booklet to each patient of the intervention group and explained the precautions and back exercises to them during the preoperative period and also postoperatively through 4 individualized sessions during hospitalization period; each session lasted about 45-60 minutes. Through these sessions, the researcher trained each patient of the intervention group individually to do back exercises by themselves.

\section{The educational booklet}

Each patient of the intervention group received preoperatively a copy of an educational booklet developed by the research team (the medical-surgical nursing researcher, the physiotherapist and the neurosurgeons). The booklet was developed guided by previously used evidence-based booklets designed for patients having surgical operation for either disc prolapse or spinal decompression. ${ }^{[12,13]}$ The research team committee agreed on the statement to be translated from the revised booklets. The selected sentences were forward translated from English into Arabic by two of the researchers, the medical-surgical nursing researcher and the physiotherapist, and another forward translation was performed by a professional translator without any medical background. The variations between the two translations had been discussed and resolved and a consensus was developed about the Arabic wording of each item. Cognitive testing of the translated version was done by open interviews with ten patients using the final translated version and asking those patients if they understood clearly each instruction. They were also asked to perform the required exercises under the researcher's observation. Very minor changes were required and performed in the final Arabic version. The booklet was revised and approved by professors of neurosurgery and physiotherapy to ensure validity of the Arabic version of the booklet in terms of the linguistic integrity of the phrases.

The booklet included detailed information about: Postoperative precautions and back exercises:

- Posture correction: Sitting and standing.

- Activities at home: Sleeping/lying position, bathing, dress- 
ing, housework/yard work, driving, leisure and recreation, returning to work and sexual activity and the use of back brace.

- Exercises which included breathing, circulation, and early stretching and strengthening exercises (buttock squeeze and abdominal tightening) performed within 0-2 weeks postoperatively.
- Stretching and strengthening exercises: buttock squeeze, abdominal tightening, knee to chest, hamstring stretch, calf stretch, partial squat, upper back strengthening, ankle strengthening and standing hip extension that started within 4-6 weeks postoperatively.

- Recommended follow up appointments are also listed in the booklet.

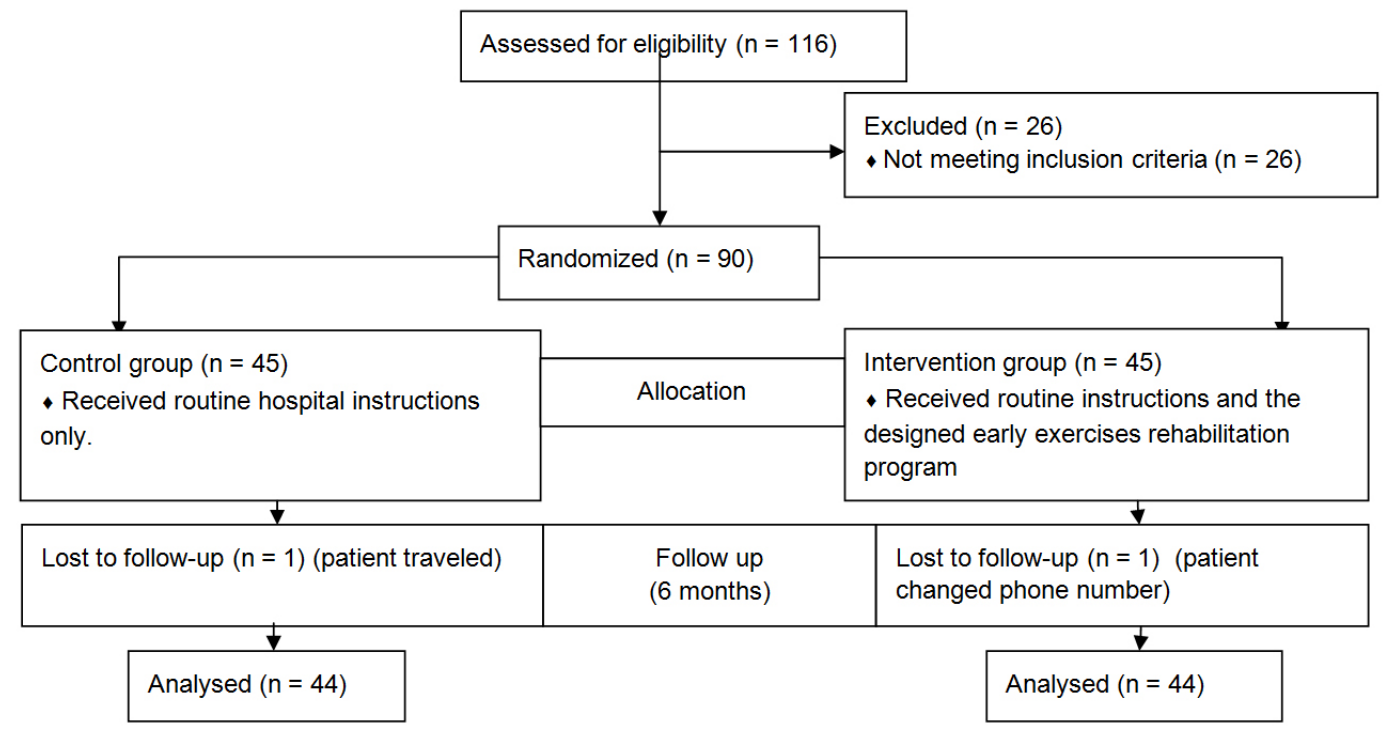

Figure 1. Flow diagram of study sample recruitment

\subsection{Data collection tools}

Patients' data were collected using assessment sheet for patients with open lumbar discectomy and Oswestry disability index (ODI).

Assessment sheet for patients with open lumbar discectomy: Patients' demographic data were recorded. Clinical examination of patients was done to assess their neurological status; reflexes, Medical Research Council Scale of Muscle Strength, ${ }^{[14]}$ muscle tone and sphincter control. Medical Research Council Scale of Muscle Strength is the most frequently used tool for evaluating muscle strength and was used in this study. This tool uses a graded scale from zero to five. A score of " 0 " indicates no muscle contraction while a score of " 5 " indicates normal muscle strength. Comorbidities and postoperative complications were recorded.

Oswestry disability index: It used to measure patient's functional level. We used a validated Arabic version of this questionnaire. ${ }^{[15,16]}$ It consists of 10 domains: pain intensity, personal care, lifting, walking, sitting, standing, sleeping, sexual life, social life and travelling. Each domain is scored from 0-5, higher values indicate greater disability. The highest possible score of ODI is 50. The results are expressed as a percentage of the maximum score (score/total score (50) * 100). Scores from 0-20 indicate minimal disability, 21-40 moderate disability, 41-60 severe disability, 61-80 crippled and 81-100 bed-bound or exaggerated symptoms.

Before discharge, follow up appointments were set with patients for the aim of observing, monitoring and evaluating their outcomes. Patients were followed up through phone calls and attendance to outpatients every two months for six months.

\subsection{Ethical considerations}

The study design was approved by a University's Research Ethical committee. An oral informed consent was obtained from all participants after a detailed explanation of the study purpose and nature.

\subsection{Statistical analysis}

Statistical analysis was done by using IBM SPSS software (version 21.0). Descriptive statistics of the studied sample was done in the form of frequencies, means and standard deviations to describe the sample characteristics and parameters of baseline clinical assessment. Baseline characteristics of both groups were compared using independent sample $t$-test for quantitative variables and chi-square test for qualitative variables to ensure matching of both groups at baseline and the non-significant differences between them before application of the early exercises rehabilitation program. The 
ODI was calculated using the 10 domains along a 5 point Likert scale. Scoring was graded from score "0" given to the least perceived disability to score " 5 " given to the severest perceived disability, with higher score reflecting more disability. All scores were summed and mean score was calculated. The ODI scores were compared at baseline and at six months postoperatively using paired $t$-test, while the difference in the scale score between control and intervention groups at six months postoperatively was assessed using independent sample $t$-test. Multivariable linear regression analysis was performed to identify the predictors of the ODI score at six months evaluation postoperatively. All the hypothesized variables to affect the independent variable; ODI score, were entered in the regression model. Statistical difference considered significant when $p$-value was less than .05 .

\section{Results}

Table 1 shows the demographic characteristics of the study participants. Control and intervention groups were matched and no significant statistical differences were found between the two groups regarding any of the demographic characteristics or baseline clinical assessment. The mean ages of intervention and control groups were $37.32 \pm 11.80$ and $39.39 \pm 11.48$ years, respectively. Just more than half of the intervention and control groups were males; $54.5 \%$ and $56.8 \%$, respectively. The majority of both groups were married. About two thirds of the study participants had lumbar 4-5 disc herniation while one third had lumbar 5 - sacral 1 disc herniation. Regarding the baseline clinical assessment, the majority of control and intervention groups had normal reflexes, power, tone and functioning urinary sphincters. Less than one fifth of the study participants were diabetics and much fewer participants were hypertensive (11.4\% of intervention and $9.1 \%$ of control groups).

Regarding the baseline assessment differences of the participants using the ODI, there were non-significant differences between the total ODI score in both groups. The mean ODI score for intervention group $9.31 \pm 6.04$ and for control group $10.93 \pm 8.13$.

Table 1. Characteristics and baseline clinical assessment of the study participants

\begin{tabular}{|c|c|c|c|c|c|}
\hline \multirow{2}{*}{ Variables } & \multicolumn{2}{|c|}{ Intervention group $(n=44)$} & \multicolumn{2}{|c|}{ Control group $(n=44)$} & \multirow{2}{*}{$p$-value } \\
\hline & No. & $\%$ & No. & $\%$ & \\
\hline \multicolumn{6}{|l|}{ Age (years) } \\
\hline Mean \pm SD & \multicolumn{2}{|c|}{$37.32 \pm 11.80$} & \multicolumn{2}{|c|}{$39.39 \pm 11.48$} & .407 \\
\hline \multicolumn{6}{|l|}{ Sex } \\
\hline Male & 24 & 54.5 & 25 & 56.8 & .830 \\
\hline Female & 20 & 45.5 & 19 & 43.2 & \\
\hline Married & 39 & 88.6 & 41 & 93.2 & .458 \\
\hline Single & 5 & 11.4 & 3 & 6.8 & \\
\hline \multicolumn{6}{|l|}{ Level of lumbar disc herniation } \\
\hline Lumbar 4-5 & 27 & 61.4 & 30 & 68.2 & .230 \\
\hline Lumbar 5-sacral1 & 17 & 38.6 & 14 & 31.8 & \\
\hline \multicolumn{6}{|l|}{ Reflexes } \\
\hline Hyporeflexia & 6 & 13.6 & 5 & 11.4 & \\
\hline \multicolumn{6}{|l|}{ Muscle power } \\
\hline Normal muscle strength & 30 & 68.2 & 31 & 70.5 & .836 \\
\hline Muscle movement against gravity with some resistance & 11 & 25.0 & 9 & 20.5 & \\
\hline Muscle movement against gravity with full resistance & 3 & 6.8 & 4 & 9.1 & \\
\hline \multicolumn{6}{|l|}{ Muscle tone } \\
\hline Normal & 40 & 90.9 & 40 & 90.9 & 1.00 \\
\hline Hypotonia & 4 & 9.1 & 4 & 9.1 & \\
\hline \multicolumn{6}{|l|}{ Sphincters } \\
\hline Normal & 37 & 84.1 & 36 & 81.8 & .956 \\
\hline Retention & 6 & 13.6 & 7 & 15.9 & \\
\hline Incontinence & 1 & 2.3 & 1 & 2.3 & \\
\hline \multicolumn{6}{|l|}{ Diabetes mellitus } \\
\hline Yes & 8 & 18.5 & 8 & 18.5 & 1.00 \\
\hline Yes & 5 & 11.4 & 4 & 9.1 & .725 \\
\hline \multicolumn{6}{|l|}{ Oswestry disability index score } \\
\hline Mean \pm SD & 9.31 & & 10.9 & & .317 \\
\hline
\end{tabular}


Table 2 shows that both groups had statistically significant improved ODI scores at six months postoperatively as compared to the baseline assessment. The mean ODI score of intervention group at six months postoperatively was 1.44 \pm 1.63 , compared to $9.31 \pm 6.04$ at baseline assessment ( $p$ $<.01$ ). Also, the mean ODI score of control group at six months postoperatively was $4.29 \pm 4.97$, compared to 10.93 \pm 8.13 at baseline assessment $(p<.01)$.

Table 2. Oswestry disability index assessment at baseline and 6 months postoperatively following early exercises rehabilitation program

\begin{tabular}{lll}
\hline & $\begin{array}{l}\text { Baseline } \\
\text { ODI score }\end{array}$ & $\begin{array}{l}\text { Oswestry disability } \\
\text { index score at } 6 \text { months } \\
\text { postoperatively }\end{array}$ \\
\hline & Mean \pm SD & Mean \pm SD \\
Intervention group $(\mathrm{n}=44)$ & $9.31 \pm 6.04^{*}$ & $1.44 \pm 1.63$ \\
Control group $(\mathrm{n}=44)$ & $10.93 \pm 8.13^{*}$ & $4.29 \pm 4.97$ \\
\hline${ }^{*} p<.01$ & &
\end{tabular}

Table 3 shows the mean scores of ODI different domains for the intervention and control groups at six months postoperatively. It was found that there was significant improvement among the intervention group as compared to the control group in several domains such as; walking, sitting, standing, sleeping, travelling and sexual and social life $(p<.01)$.
Table 3. Oswestry disability index scores of intervention and control groups at 6 months evaluation postoperatively

\begin{tabular}{lll}
\hline $\begin{array}{l}\text { Oswestry disability } \\
\text { index domains }\end{array}$ & $\begin{array}{l}\text { Intervention group } \\
(\mathbf{n}=\mathbf{4 4 )}\end{array}$ & $\begin{array}{l}\text { Control group } \\
(\mathbf{n}=\mathbf{4 4 )}\end{array}$ \\
\cline { 2 - 3 } & Mean $\pm \mathbf{S D}$ & Mean \pm SD \\
\hline Pain intensity & $0.41 \pm 0.54$ & $0.50 \pm 0.63$ \\
Personal care & $0.41 \pm 0.49$ & $0.32 \pm 0.56$ \\
Lifting & $0.41 \pm 0.58$ & $0.50 \pm 0.73$ \\
Walking & $0.00 \pm 0.00^{*}$ & $0.34 \pm 0.57$ \\
Sitting & $0.50 \pm 0.30^{*}$ & $0.45 \pm 0.59$ \\
Standing & $0.00 \pm 0.00^{*}$ & $0.39 \pm 0.62$ \\
Sleeping & $0.00 \pm 0.00^{*}$ & $0.39 \pm 0.57$ \\
Sexual life & $0.00 \pm 0.00^{*}$ & $0.49 \pm 0.78$ \\
Social life & $0.00 \pm 0.00^{*}$ & $0.48 \pm 0.66$ \\
Travelling & $0.09 \pm 0.29^{*}$ & $0.45 \pm 0.59$ \\
Total score & $1.44 \pm 1.63$ & $4.29 \pm 4.97$ \\
\hline${ }^{*} p<.01$ & &
\end{tabular}

Table 4 shows the predictors of better ODI score at six months evaluation postoperatively; it was found that lower ODI score at baseline predicted lower ODI score at six months postoperatively $(\beta=0.242$, Standard error $=0.053$, $p$-value $<.001)$. Moreover, receiving the early exercises rehabilitation program and educational booklet predicted better ODI score at the six months evaluation postoperatively ( $\beta=$ 2.506 , Standard error $=0.752, p$-value $<.01$ ).

Table 4. Predictors of better ODI score at the 6 months evaluation postoperatively

\begin{tabular}{llll}
\hline Predictors & $\begin{array}{l}\text { Unstandardized B } \\
\text { coefficient }\end{array}$ & Standard error & $\begin{array}{l}\text { 95\% Confidence } \\
\text { interval for B }\end{array}$ \\
\hline Age & -0.018 & 0.033 & $-0.084-0.048$ \\
Sex & 0.747 & 0.760 & $-0.768-2.262$ \\
Level of lumbar disc herniation & 0.426 & 0.632 & $-0.834-1.686$ \\
$\begin{array}{l}\text { Baseline ODI score } \\
\text { Received early exercises rehabilitation program and a } \\
\text { designed educational booklet }\end{array}$ & 0.242 & 0.053 & $0.137-0.348$ \\
\hline
\end{tabular}
$* p<.01 ; * * p<.001$.

\section{Discussion}

In the present study, comparison of the baseline characteristics of patients in the intervention and control groups showed that both groups were similar. In the first measurement (before starting the early exercises rehabilitation program), the mean scores obtained from the ODI by the patients in the intervention and control groups were close to each other, and there was non significant difference between them. These results are important in that patients in both groups had similar scores and characteristics. It was determined that the ODI scores of patients in both groups were high (high ODI score indicating high level of disability). This result is similar to the results of Azimi et al. (2016) who conducted a study Published by Sciedu Press on 154 patients after discectomy and stated that the baseline ODI scores was high due to pain and difficult in performing physical functions. ${ }^{[17]}$

In our study, there was difference in the two groups in terms of final measurements. The early exercises rehabilitation program which was applied in the intervention group significantly decreased pain, difficult in performing physical functions and total ODI scores. The early exercises rehabilitation program was effective in improving patients' outcomes.

Clinical trial data demonstrate improved outcomes after postoperative early exercise rehabilitation programs, suggesting that treatment should be initiated sooner to predict improved outcomes. $^{[18,19]}$ Ostelo et al. (2009), demonstrated that 
current best practices encourage the use of a rehabilitation program after lumbar discectomy. However, there are no widely accepted criteria to what constitutes the most favorable rehabilitation program. ${ }^{[20]}$

We found that both groups experienced high score of ODI at baseline assessment. Intervention group had statistically significant improved scores of ODI at six months postoperatively compared to the control group due to the identified predictors (i.e. better scores at baseline and the received early exercises rehabilitation program using educational booklet).

Despite the significant improvement of ODI score for both groups at six months postoperatively as compared to the baseline assessment, patients in the intervention group benefitted from the early exercises rehabilitation program using educational booklet while patients of control group still experienced problems with everyday functioning because of back and leg pain.

These findings were similar to those found in a study conducted by Hebert et al. (2010), who reported that patient experienced clinical improvements in both pain and disability post-application of a rehabilitation program after lumbar discectomy. ${ }^{[21]}$

In our study, better baseline ODI scores predicted better ODI scores (pain improvement) at six months postoperatively. Significant improvements were observed in some dimensions such as: walking, sitting, standing, sleeping, travelling, and sexual and social life. Demographic variables (age and sex) and level of lumbar disc herniation were not significantly associated with better ODI scores at six months postoperatively.

Our findings support the results of a study conducted by Azimi and Benzel (2017), in which they reported that significant improvements in ODI scores were observed between baseline (37.7 \pm 14.8$)$ and follow up $(16.2 \pm 11.7) .{ }^{[22]}$ Similarly, Soriano et al. (2010), also found that age and sex had no predictive value in all outcome measure. ${ }^{23]}$

A study by Rushton et al. (2018), identified that there was a very low-quality evidence that pre-operative ODI predict better ODI at twelve months. Lumbar discectomy success rates have been reported as high as $78 \%-95 \%$ at one to two years post-surgery. However, continuing back and leg pain and disability are issues for some patients. It is important to recognize prognostic factors predicting patients' outcomes to inform decision-making for rehabilitation following lumbar discectomy. ${ }^{[24]}$

Our study was inconsistent with the study of Soriano et al. (2010), which concluded that intensity of back pain had a neg- ative association with patients' improvements. Severe preoperative back/leg pain predicted lower postoperative ODI scores. ${ }^{[23]}$

Patient education is fundamentally the most important role of the nurse. To achieve better patients' outcomes, continuing care by nurses should be provided to be able to play their professional role in their care. Continuing care is a process, which is regularly conducted with goal of making an efficient nurse-patient communication and interaction among rehabilitation nurses, as the health care providers, to detect patients' needs and problems to facilitate preservation, recovery, and improvement of patients' care. ${ }^{[25]}$

The findings indicated that an early exercises rehabilitation program using educational booklet improves outcomes of patients following open lumbar discectomy. Nurses can teach, train and motive patients following open lumbar discectomy to perform and adhere to the exercises rehabilitation program.

\subsection{Limitations}

Our study had some limitations. The small sample size and the short study follow up may not be adequate to evaluate the long term effect of the early exercises rehabilitation program and the predictive factors for improvement.

\subsection{Key practice points}

- Comprehensive post lumbar discectomy rehabilitation program should include early exercises.

- It is essential for the rehabilitation nurse to promote early exercises.

- Early exercises rehabilitation program decreased both pain and difficult in performing physical functions.

- Application of the early exercises rehabilitation program using an educational booklet predicted better ODI score (pain improvement) during follow up period.

\subsection{Implications for rehabilitation nursing}

As our findings have proved that an early exercises rehabilitation program using an educational booklet was useful and effective in improving the postoperative functional outcomes of patients, we recommend distributing the translated Arabic version of the educational booklet in the neurosurgery departments in different hospitals in Egypt to be a guiding tool for both nurses and patients about the recommended physical exercises following lumbar discectomy. Using the designed intervention could be helpful to support independent living at home and improve patients' quality of life.

Our study findings are expected to predict the effect of an early exercises rehabilitation program aiming to eliminate or control pain, difficult in performing physical functions which considered the most common problems following open 
lumbar discectomy and determine the predictive factors of improvement. The results are also expected to encourage nurses to utilize an early exercises rehabilitation program when providing nursing care.

\section{Conclusion}

Our results showed that an early exercises rehabilitation program using an educational booklet significantly improves postoperative pain of patients with single level open lumbar discectomy. More severe preoperative back pain had less favorable postoperative outcomes at six months postopera- tively. Nurses should assess pain and difficult in performing physical functioning for patients, which are important issues in the care of patients following open lumbar discectomy; utilize early exercises rehabilitation program to solve these problems and encourage patients to do early exercises rehabilitation program. Better ODI score at baseline and the received early exercises rehabilitation program and educational booklet predicted better ODI score (pain improvement) during follow up period.

\section{CONFLiCTS OF INTEREST Disclosure}

The authors declare that there is no conflict of interest.

\section{REFERENCES}

[1] Buchbinder RBF, March LM. Placing the global burden of low back pain in context. Best Pract Res ClinRheumatol. 2013; 27(5): 575-89. PMid:24315140 https : //doi.org/10.1016/j.berh.2013.10 .007

[2] Malik KM, Jabri RS, Benzon HT. Overview of Low Back Pain Disorders, Chapter 43, Elsevier Inc.; 2011; 294-306. PMid:21913587 https : //doi.org/10.1016/B978-1-4377-2242-0.00052-3

[3] Abouelela AA, Morsi AM, Khattab MF. Microscopic lumbar discectomy. The Egyptian Orthopaedic Journal. 2015; 50(1): 15. https://doi.org/10.4103/1110-1148.163115

[4] Ullrich PF. Lumbar Spine Surgery, Published article. 2013. Available from: http://www.spinehealth.com/treatment/back -surgery/lumbar-spine-surgery

[5] Kanaan S. Predicting Health Care Needs Following Lumbar Spine Surgery, Doctoral dissertation, University of Kansas. 2013.

[6] McFarland CM. Rehabilitation and Exercise Following Spine Surgery. Published article. 2015. Available from: http://www.spine-health.com/wellness/exercise/reha bilitation-and-exercise-following-spine-surgery

[7] Association of Rehabilitation Nurses. ARN Position Statement-Role of the Nurse in the Rehabilitation Team. Chicago, IL: Author. 2014.

[8] Ozkara GO, Ozgen M, Ozkara E, et al. Effectiveness of physical therapy and rehabilitation programs starting immediately after lumbar disc surgery. Turkish Neurosurgery. 2015; 25(3): 372-379.

[9] Lonne G, Solberg TK, Sjaavik K, et al. Recovery of muscle strength after microdiscectomy for lumbar disc herniation: a prospective cohort study with 1-year follow-up. European Spine Journal. 2012; 21(4): 655-659. PMid:22193841 https ://doi.org/10.1007/s0 0586-011-2122-x

[10] Poppert EM, Kulig K. Rehabilitation following lumbar discectomy. Physical Therapy. 2013; 93(5): 591-596. PMid:23449913 https://doi.org/10.2522/ptj. 20120087

[11] Kjaer P, Kongsted A, Ris I, et al. GLA: D Back group-based patient education integrated with exercises to support self-management of back pain-development, theories and scientific evidence. BMC Musculoskeletal Disorders. 2018; 19(1): 418. PMid:30497440 https : //doi.org/10.1186/s12891-018-2334-x

[12] Saskatchewan Spine Pathway Working Group. Back surgery: a guide for patients and their coaches. 2015; 8-38.

[13] Johns Hopkins Spine Service. The road to recovery after lumbar spine surgery, A detailed guide to your surgery and the recovery process. Orthopedic Surgery. 2007; 13-27.
[14] Van der Ploeg RJO, Oosterhuis HJGH, Reuvekamp J. Measuring muscle strength. Journal of Neurology. 1984; 231(4): 200-203. PMid:6512574 https ://doi.org/10.1007/BF00313939

[15] Fairbank JC, Pynsent PB. The Oswestry disability index. Spine. 2000; 25(22): 2940-2953. PMid:11074683 https://doi.org/10.109 7/00007632-200011150-00017

[16] Algarni AS, Ghorbel S, Jones JG, et al. Validation of an Arabic version of the Oswestry index in Saudi Arabia. Annals of Physical and Rehabilitation Medicine. 2014; 57(9-10): 653-663. PMid:25262247 https://doi.org/10.1016/j.rehab.2014.06.006

[17] Azimi P, Benzel EC, Montazeri A. Predictive score card in lumbar disc herniation: Is it reflective of patient surgical success after discectomy?. PloS One. 2016; 11(4): p.e0154114. PMid:27100287 https://doi.org/10.1371/journal.pone.0154114

[18] Kulig K, Beneck GJ, Selkowitz DM. An intensive, progressive exercise program reduces disability and improves functional performance in patients after single-level lumbar microdiscectomy. Physical Therapy. 2009; 89: 1145-1157. PMid:19778981 https : //doi.org/10.2522/ptj. 20080052

[19] Millisdotter M, Stromqvist B. Early neuromuscular customized training after surgery for lumbar disc herniation: a prospective controlled study. European Spine Journal. 2007; 16: 19-26. PMid:16421747 https : //doi .org/10.1007/s00586-005-0044-1

[20] Ostelo RW, Costa LOP, Maher CG, et al. Rehabilitation after lumbar disc surgery: an update Cochrane review. Spine. 2009; 34(17): 18391848. PMid:19602996 https ://doi.org/10.1097/BRS. Ob013e 3181abbfdf

[21] Hebert JJ, Marcus RL, Koppenhaver SL, et al. Postoperative rehabilitation following lumbar discectomy with quantification of trunk muscle morphology and function: a case report and review of the literature. Journal of Orthopedic and Sports Physical Therapy. 2010; 40(7): 402-412. PMid:20592478 https://doi .org/10.2519/jo spt. 2010.3332

[22] Azimi P, Benzel EC. The Low-Back Outcome Scale and the Oswestry disability index: are they reflective of patient satisfaction after discectomy? A cross sectional study. Journal of Spine Surgery. 2017; 3(4): 554. PMid:29354731 https://doi.org/10.21037/jss.2 017.09 .07

[23] Soriano JC, Revuelta MS, Fuente MF, et al. Predictors of outcome after decompressive lumbar surgery and instrumented posterolateral fusion. European Spine Journal. 2010; 19(11): 1841-1848. PMid:20135333 https://doi.org/10.1007/s00586-010-128 4-2

Published by Sciedu Press 
[24] Rushton A, Zoulas K, Powell A, et al. Physical prognostic factors predicting outcome following lumbar discectomy surgery: systematic review and narrative synthesis. BMC Musculoskeletal Disorders. 2018; 19(1): 326. PMid:30205812 https://doi.org/10.1186/ s12891-018-2240-2
[25] Alimohammadi N, Eslami M, Yousefi $\mathrm{H}$, et al. The effect of continuing care on patient's quality-of-life after disc surgery in neurosurgery and very important person wards. Journal of Education and Health Promotion. 2015; 4(106): 1-6. 